\title{
An Atmospheric Correction Algorithm for FY3/MERSI data over land in China
}

\author{
Cheng Fan ${ }^{1,3}$, Jie Guang ${ }^{1}$, Yong Xue ${ }^{1,2 *}$, Aojie Di ${ }^{1,3}$, Lu She ${ }^{1,3}$, Yahui Che $e^{1,3}$ \\ ${ }^{1}$ State Key Laboratory of Remote Sensing Science, Institute of Remote Sensing and Digital Earth, \\ Chinese Academy of Sciences, Beijing 100101, China \\ ${ }^{2}$ Faculty of Life Sciences and Computing, London Metropolitan University, 166-220 Holloway Road, \\ London \\ N7 8DB, UK \\ ${ }^{3}$ University of Chinese Academy of Sciences, Beijing 100049, China \\ \{Email:chengfjane@163.com,yx9@hotmail.com\}
}

\begin{abstract}
Feng-Yun (FY-3) is the second generation of the Chinese Polar Orbiting Meteorological Satellites with global, three-dimensional, quantitative, and multispectral capabilities. Medium Resolution Spectral Imager (MERSI) has 20 channels onboard the FY-3A and FY-3B satellites, including five channels (four VIS and one thermal IR) with a spatial resolution of $250 \mathrm{~m}$. The top of the atmosphere signal are necessary to be radiometrically calibrated and corrected for atmospheric effects based on surface reflectance, especially in land surface remote sensing and applications. This paper presents an atmospheric correction algorithm for FY3/MERSI data over land in China, taking into account the directional properties of the observed surface by a kernel-based Bi-directional Reflectance Distribution Function (BRDF) model. The comparison with MODGA and ASD reflectance showed that there is a good agreement. Therefore, FY3/MERSI can serve a reliable and new data source for quantifying global environment change.
\end{abstract}

Index Terms - MERSI, Surface Reflectance, Aerosol Optical Depth

\section{INTRODUCTION}

Surface reflectance is a necessary parameter for many applications of Earth-observing satellite radiometer measurements in the visible and near infrared band, including derivation of various geographical parameters. The atmospheric effects need to be removed based on surface reflectance, especially in land surface remote sensing and application. Surface reflectance retrieval is an important step in the data processing chain for the extraction of quantitative information in many applications [1]. Atmospheric aerosol particles play an important role in the Earth's radiation balance. They scatter and absorb solar radiation (direct effect) and affect the albedo and lifetime of clouds (indirect effect). In spite of different Aerosol Optical depth (AOD) retrieval algorithms have been established over land for different sensors, most of the retrieval algorithms are based on surface reflectance and have assumed a Lambertian surface. This has allowed the development of the algorithms to retrieval AOD and surface reflectance simultaneously over both dark vegetated surfaces and bright land surface.

Feng-Yun (FY-3) is the second generation of the Chinese Polar Orbiting Meteorological Satellites. Medium Resolution Spectral Imager (MERSI) is one of the sensors onboard FY3A and FY3B, which are both sun-synchronous polar orbit satellites. Atmospheric correction of visible-infrared band FY3A/MERSI data based on $6 \mathrm{~S}$ model has been proposed [2]. FY-3A/MERSI data was used to study the oil spill detection on the basis of the Quick Atmospheric Correction model (QUAC) in ENVI software to remove the atmospheric effect [3]. MERSI data has the great potential for global LAI retrieval by comparing with MODIS data [4]. Sun found a new vicarious calibration (VC) method for RSBs based on in-situ BRDF model and vector radiometric transfer model 6SV with gaseous absorption correction using MODTRAN [5]. Liu [6] proposed a new atmospheric correction method named Single Band Water Vapor Dependent (SBWVD) method for LST retrieval from FY3A/MERSI with only one thermal infrared channel. However, little attention has been paid to surface reflectance using FY3/MERSI data.

In this paper, the potentiality of the retrieval of surface reflectance using FY3/MERSI data was studied. We present an atmospheric correction algorithm SARBA (a Synergistic Approach for Retrieving BRDF and Aerosol) for both FY3A and 
FY3B MERSI in visible to near-infrared band over land in China. Previous operational correction schemes have assumed a Lambertian surface. This atmospheric correction algorithm is developed to take into account of the directional properties of the observed surface by a kernel-based Bi-directional Reflectance Distribution Function (BRDF) model. We built a series of equations to retrieve both surface reflectance and AOD at the same time. In this paper, we applied this method to FY3A and FY3B MERSI for retrieving surface reflectance over land in China.

\section{MODELS}

A novel model SARBA (a Synergistic Approach for Retrieving BRDF and Aerosol) using both FY3A/MERSI and FY3B/MERSI data was proposed. This method has already successfully applied to Terra/MODIS and Aqua/MODIS data [1, 7].

Substituting the exact integrodifferential equation for radiant intensity by common differential equations for the upward and incident radiation fluxes is the main idea of the deduction of the most frequently used approximation transfer equations. The general solution of this problem has been given by Kuznetzov[8].Therefore we can find the relation between the ground surface reflectance $A$ and apparent reflectance (reflectance on the top of atmosphere) $A^{\prime}$, which is proposed by Xue and Cracknell[9] as follows:

$$
A=\frac{\left(A^{\prime} b-a\right)+a\left(1-A^{\prime}\right) e^{(a-b) \varepsilon \tau_{0}^{\lambda} \sec \theta^{\prime}}}{\left(A^{\prime} b-a\right)+b\left(1-A^{\prime}\right) e^{(a-b) \varepsilon \tau_{0}^{\lambda} \sec \theta^{\prime}}}
$$

Where $a=\sec \theta$ and $b=2, \varepsilon$ is the backscattering coefficient, typically 0.1 .The solar zenith angle is calculated from latitude, longitude and satellite pass time or the data set for MERSI. The atmospheric optical depth $\tau_{0}^{\lambda}$ is determined by the atmospheric turbidity state of passing time.

For our model SARBA, only taking account of the scattering of atmospheric molecular and aerosol particles, we assume the atmospheric optical depth $\tau_{0}^{\lambda}$ consisting of two parts: the molecular Rayleigh scattering $\tau_{M}^{\lambda}(\infty)$ and the scattering of aerosol particles $\tau_{A}^{\lambda}(\infty)$. Therefore the dimensionless quantity of the optical depth of the whole atmosphere is as follows:

$$
\tau_{0}^{\lambda}=\tau_{M}^{\lambda}(\infty)+\tau_{A}^{\lambda}(\infty)
$$

For the molecular Rayleigh scattering $\tau_{M}^{\lambda}(\infty)$, Linke has given an approximate expression which is sufficiently accurate for most application in remote sensing as follows:

$$
\tau_{M}^{\lambda}(\infty)=0.00879 \lambda^{-4.09}
$$

For the scattering of aerosol particles $\tau_{M}^{\lambda}(\infty)$, we take Angstrom's turbidity formula as follows:

$$
\tau_{A}^{\lambda}(\infty)=\beta \lambda^{-\alpha}
$$

Substituting eq. (4), (3) and (2) into eq. (1), we can obtain a new equation on the relation among the parameters of ground surface reflectance, Angstrom's turbidity coefficient $\beta$ and wavelength exponent $\alpha$. Other parameters are from the data set of satellite images and the satellite-earth-sun geometric information.

Now, if we substitute bi-temporal satellite data such as three visible spectral bans data, central wavelength of $470 \mathrm{~nm}$, $550 \mathrm{~nm}, 650 \mathrm{~nm}$, respectively from FY3A and FY3 MERSI into eq.(1), we can obtain one group of nonlinear equation as follows:

$$
A_{j, \lambda_{i}}=\frac{\left(A_{j, \lambda_{i}}^{\prime} b_{j}-a_{j}\right)+a_{j}\left(1-A_{j, \lambda_{i}}^{\prime}\right) e^{\left(a_{j}-b_{j}\right) \varepsilon\left(0.00879 \lambda_{i}^{-4.09}+\beta_{j} \lambda_{i}^{-\alpha}\right) \sec \theta_{v, j}^{\prime}}}{\left(A_{j, \lambda_{i}}^{\prime} b_{j}-a_{j}\right)+b_{j}\left(1-A_{j, \lambda_{i}}^{\prime}\right) e^{\left(a_{j}-b_{j}\right) \varepsilon\left(0.00879 \lambda_{i}^{-4.09}+\beta_{j} \lambda_{i}^{\alpha}\right) \sec \theta_{v, j}^{\prime}}}
$$

Where $j=1,2$, respectively stand for the observation of FY3A and FY3B MERSI; $i=1,2,3$, respectively stand for three visible spectral bands of central wavelength of $470 \mathrm{~nm}$, $550 \mathrm{~nm}, 660 \mathrm{~nm} ; \lambda$ is the central wavelength.

We also used the semiemirical, kernel-based Ross-Li BRDF model[10], to correct reflectance anisotropy effects. The Ross-Li model allows decomposing measured HDRF into radiation components originating from three surface scattering types. Isotropic scattering (ISO) is the radiation fraction without angular dependence. Volumetric scattering (VOL) has angular dependence as a function of small inter-leaf gaps within a horizontally homogenous vegetation canopy. Geometric scattering (GEO) generates angular dependence as function of large gaps within a canopy or sparse stand structure [11]. $K_{v o l}$ and $K_{\text {geo }}$ are nonlinear functions (so-called "kernel" $\mathrm{K}$ ) of observation and illumination geometry only. The kernels themselves are linearly combined, using a waveband specific weighting parameter $f$. This weighting parameter is retrieved by model inversion. Considering the mentioned components, the reflectance $A_{j, \lambda_{i}}$ can be then described as follow:

$$
\begin{gathered}
A_{j, \lambda_{i}}=f_{i s o}\left(\lambda_{i}\right)+f_{v o l}\left(\lambda_{i}\right) K_{v o l}\left(\theta_{s, j}, \theta_{v, j}, \phi_{j}\right)+f_{g e o}\left(\lambda_{i}\right) K_{g e o}\left(\theta_{s, j}, \theta_{v, j}, \phi_{j}\right) \\
f_{m}\left(\lambda_{n}\right)=a_{m n} f_{m}\left(\lambda_{0.65}\right)+b_{m n}
\end{gathered}
$$

Equation (7) is defined the relationship between BRDF parameters on different wavelengths to reduce the number of unknown parameters.

\section{DATA SET}

FY3A was lunched on May 27, 2008, which flies northwards passing the equator at local time 10:05 AM. FY3B lunched on Nov. 5, 2010, which flies southwards passing the equator at local time 13:00 PM. MERSI is one of the sensors on board FY3A and FY3B, which are both sun-synchronous polar orbit satellites.

The research area in this paper is mainland of China, which covers the land area between $15^{\circ} \mathrm{N}-54^{\circ} \mathrm{N}$ and $73^{\circ} \mathrm{E}-136^{\circ} \mathrm{E}$. The remote sensing data we used in this paper consist of MERSI L1B data and MERSI cloud product data with a spatial of $1 \mathrm{~km}$ of FY3A and FY3B from December 1st-31th, 2013, which were downloaded from NSMC (National Satellite Meteorological Center, China Meteorological Administration) http://satellite.nsmc.org.cn/PortalSite/default.aspx.

National Centers for Environmental Prediction (NCEP) data is also need to correct the effect of gas absorption correction on apparent reflectance and eliminate gas absorption effect of $\mathrm{H}_{2} \mathrm{O}$, $\mathrm{CO}_{2}$ and $\mathrm{O}_{3}$. NCEP data download from 
http://oceandata.sci.gsfc.nasa.gov/Ancillary/Meteorol ogical.

\section{RESULTS AND VALIDATION}

To estimate the accuracy of this method, we compared the surface reflectance results with MOD09GA and ASD reflectance. The retrieved AOD results are also compared with AERONET. The comparison of them shows that there is a good agreement. Retrieved surface reflectance on December 2nd, 2013 over China, using SARBA method is shown here in Figures 1. Figure 2 is the false color composite image by median of the surface reflectance of FY3B/MERSI band 4, 3, and 2 over China in December, 2013. Figure 3 shows comparison of the retrieved surface reflectance from MERSI and ASD Field Spec spectral radiometer measurements of band 2, with RMSE is lower than 0.1. Figure 4 shows that the comparison of MOD09GA (directional reflectance from atmospheric correction) with predicted surface reflectance from FY3/MERSI. The RMS error is lower than 0.2.

Retrieved AOD shown in Figures 5 indicates that the SARBA produce AOD values over great areas. The retrieved AOD in the present study was compared with the AOD measured at several AERONET stations. Figure 6 shows the comparisons of the retrieved AOD using SARBA method and AOD from AERONET. Since there is no value for $550 \mathrm{~nm}$ in AERONET observation values, we first estimate AOD at $550 \mathrm{~nm}$ using $870 \mathrm{~nm}$ $675 \mathrm{~nm}$ and $440 \mathrm{~nm}$. The real line stands for the regression line using SARBA method and the correlation is of 0.68 and the RMSE is about 0.3 .

\section{CONCLUSION}

A new aerosol optical depth and surface reflectance retrieved model proposed in this paper showed promising potential to address the aerosol retrieval and surface reflectance over higher reflective surface over land. It will provide us a more details about the aerosols over land by considering the surface $\mathrm{BRDF}$ effect while retrieving $\mathrm{AOD}$ and surface reflectance simultaneously. The comparison with MOD09GA and ASD reflectance showed that there is a good agreement. And the AOD retrieved by SARBA also covers a great area. Furthermore, spatial resolution of MERSI is superior as compared to that of MODIS. Therefore, FY3/MERSI can serve a reliable and new data source for quantifying global environment change.

\section{ACKNOWLEDGMENTS}

This work was supported by the Ministry of Science and Technology (MOT), China under grant Nos. 2013AA12801, and
2013CB733403. The FY3A and FY3B data was provided by China Meteorological Administration (CMA) and the validation data come from AERONET. The authors thank the staff of CMA and AERONET.

\section{REFERENCES}

[1] J. Guang, Y. Xue, L. K. Yang, L. L. Mei, and X. W. He, "A Method for Retrieving Land Surface Reflectance Using MODIS Data," Ieee Journal of Selected Topics in Applied Earth Observations and Remote Sensing, vol. 6, pp. 15641570, Jun 2013.

[2] W. U. Yongli, L. Qing, and T. Guozhen, "Atmospheric correction of visible-infrared band FY-3A/MERSI data based on 6S model," The Journal of Applied Ecology, vol. 22, pp. 1537-1542, 2011.

[3] Q. Jiang and C. Zhao, "The improvement of oil spill detection by atmospheric correction of FY-3A/MERSI data," Transactions of Oceanology and Limnology, pp. 16-24, 2011.

[4] L. Zhu, J. M. Chen, S. H. Tang, G. C. Li, and Z. D. Guo, "InterComparison and Validation of the FY-3A/MERSI LAI Product Over Mainland China," Ieee Journal of Selected Topics in Applied Earth Observations and Remote Sensing, vol. 7, pp. 458-468, Feb 2014

[5] L. Sun, M. H. Guo, N. Xu, L. J. Zhang, J. J. Liu, X. Q. Hu, et al., "On-Orbit Response Variation Analysis of FY-3 MERSI Reflective Solar Bands Based on Dunhuang Site Calibration," Spectroscopy and Spectral Analysis, vol. 32, pp. 1869-1877, Jul 2012.

[6] H. L. Liu, L. S. Xu, J. L. Ding, Basang, and X. B. Deng, "An Atmospheric Correction Method for Medium Resolution Spectral Imager Thermal IR Soundings," Advanced Materials in Microwaves and Optics, vol. 500, pp. 397-402, 2012.

[7] J. Guang, Y. Xue, Y. Li, S. Liang, L. Mei, and H. Xu, "Retrieval of aerosol optical depth over bright land surfaces by coupling bidirectional reflectance distribution function model and aerosol retrieval model," Remote Sensing Letters, vol. 3, pp. 577-584, 20122012.

[8] K. I. A. Kondrat'ev, Radiation in the atmosphere. New York,: Academic Press, 1969.

[9] Y. Xue and A. P. Cracknell, "Operational bi-angle approach to retrieve the earth surface albedo from AVHRR data in the visible band," International Journal of Remote Sensing, vol. 16, pp. 417-429, Feb 1995.

[10] J. L. Roujean, M. Leroy, and P. Y. Deschamps, "A Bidirectional Reflectance Model of the Earths Surface for the Correction of Remote-Sensing Data," Journal of Geophysical Research-Atmospheres, vol. 97, pp. 20455-20468, Dec 20 1992.

[11] X. W. Li and A. H. Strahler, "Geometric-optical bidirectional reflectance modeling of the discrete crown vegetation canopyeffect of croen shape and mutual shadowing," Ieee Transactions on Geoscience and Remote Sensing, vol. 30, pp. 276-292, Mar 1992. 

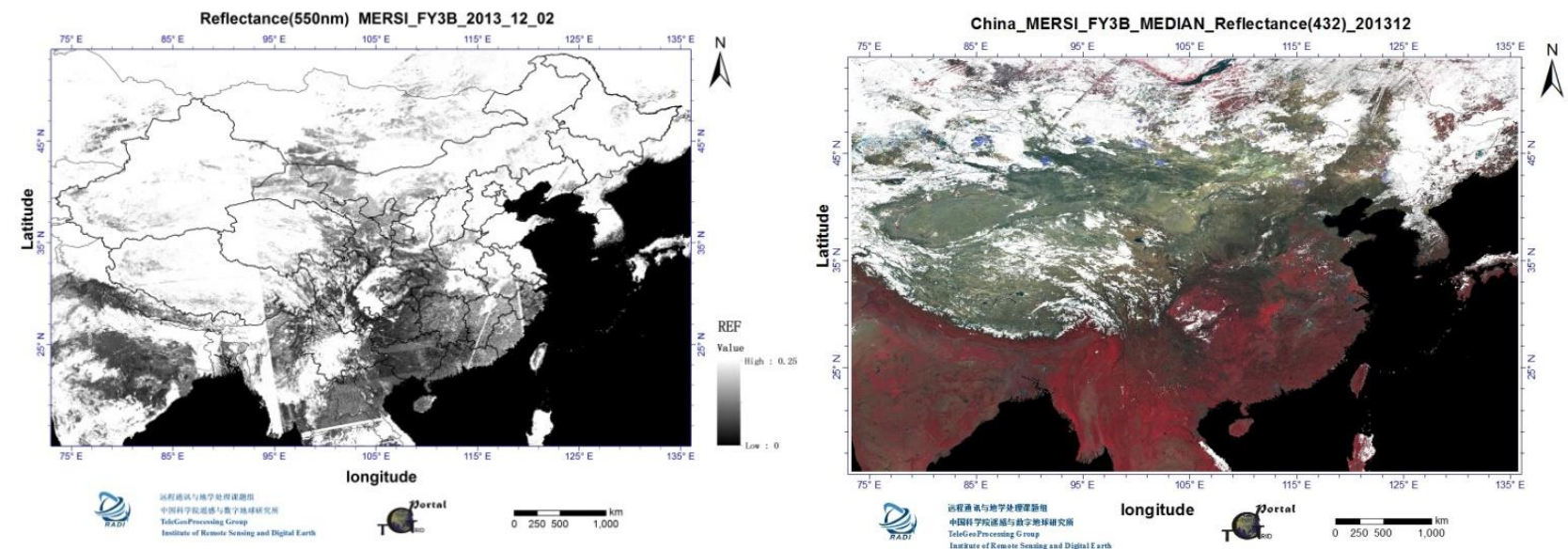

Figure1. Retrieved Surface Reflectance (550nm) of FY3B/MERSI on December 2nd, 2013, using SARBA method.

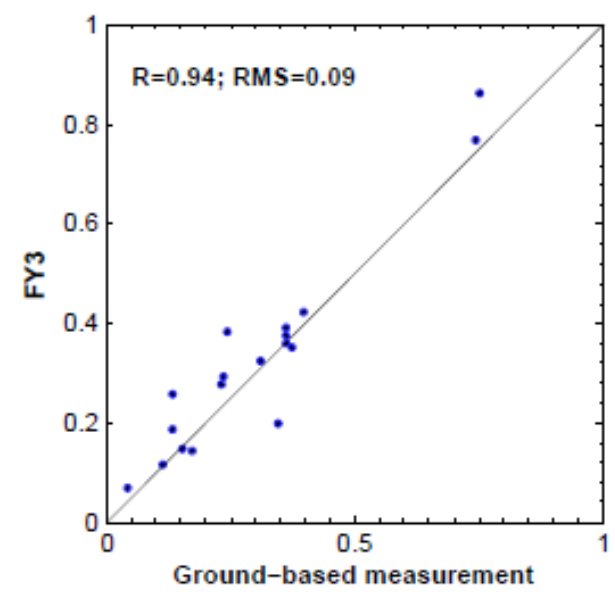

Figure 3.Comparisons of the retrieved surface reflectance from MERSI and ASD Field Spec spectral radiometer measurements of band 2 .

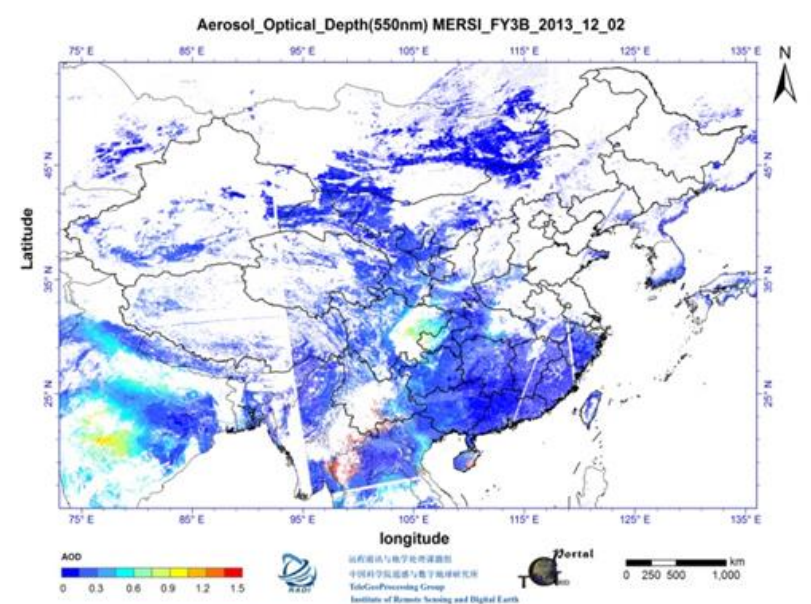

Figure 5. Retrieved AOD (550nm) on December 2nd, 2013 over China, using SARBA method.

Figure 2. False color composite image by median of the surface reflectance of FY3B/MERSI band 4, 3, and 2 over China in December, 2013.

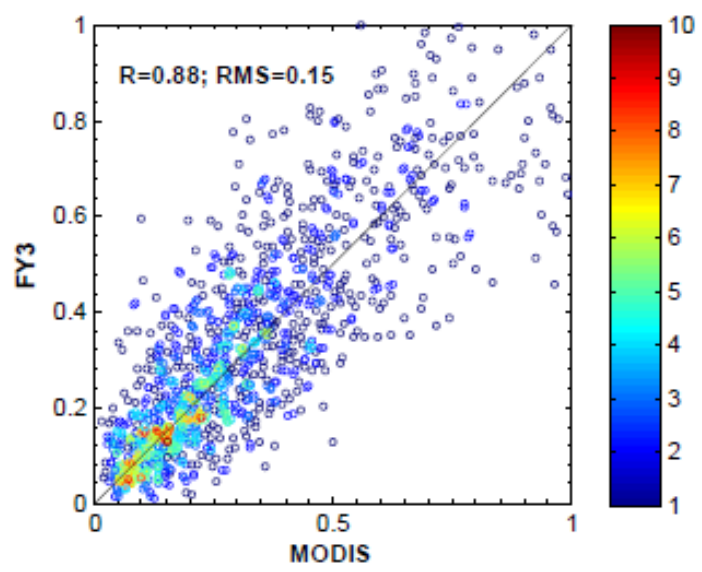

Figure 4. The comparison of MOD09GA (directional reflectance from atmospheric correction) with predicted surface reflectance $(550 \mathrm{~nm})$ from FY3/MERSI on 1 December 2013.

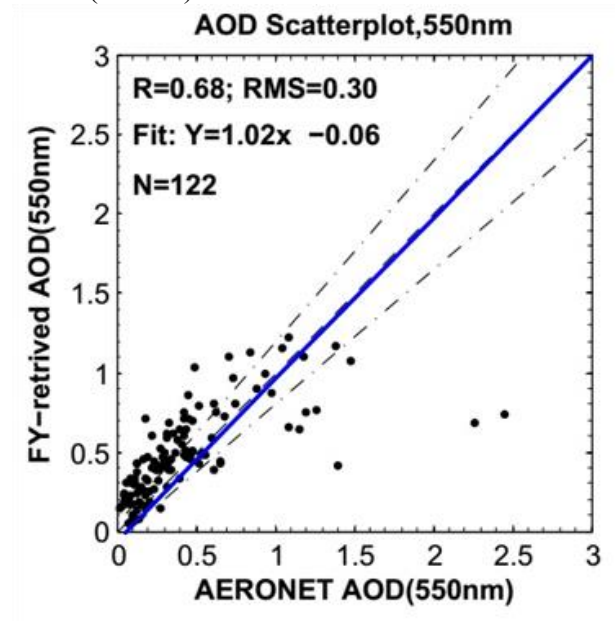

Figure 6. Comparisons of the retrieved AOD (550nm) using SARBA method and AOD from AERONET. 